\title{
Structural and immunocytochemical studies on $\alpha$-N-acetylgalactosaminidase deficiency (Schindler/Kanzaki disease)
}

Received: 29 July 2003 / Accepted: 30 September 2003/Published online: 19 December 2003

(C) The Japan Society of Human Genetics and Springer-Verlag 2003

\begin{abstract}
N$-Acetylgalactosaminidase ( $\alpha$-NAGA) deficiency (Schindler/Kanzaki disease) is a clinically and pathologically heterogeneous genetic disease with a wide spectrum including an early onset neuroaxonal dystrophy (Schindler disease) and late onset angiokeratoma corporis diffusum (Kanzaki disease). In $\alpha$-NAGA deficiency, there are discrepancies between the genotype and phenotype, and also between urinary excretion products (sialyl glycoconjugates) and a theoretical accumulated material (Tn-antigen; Gal NAc $\alpha 1-O$-Ser/Thr) resulting from a defect in $\alpha$-NAGA. As for the former issue, previously reported genetic, biochemical and pathological data raise the question whether or not E325K mutation found in Schindler disease patients really leads to the severe phenotype of $\alpha$-NAGA deficiency. The latter issue leads to the question of whether $\alpha$-NAGA
\end{abstract}

H. Sakuraba $(\square) \cdot$ M. Kotani

Department of Clinical Genetics,

The Tokyo Metropolitan Institute of Medical Science,

Tokyo Metropolitan Organization for Medical Research,

3-18-22 Honkomagome, Bunkyo-ku,

Tokyo 113-8613, Japan

E-mail: sakuraba@rinshoken.or.jp

Tel.: + 81-3-38232105

Fax: $+81-3-38236008$

F. Matsuzawa $\cdot$ S. Aikawa $\cdot$ H. Doi

Celestar Lexico-Sciences,

MTG D-17, 1-3 Nakase,

Mihama-ku, Chiba 261-8501, Japan

H. Nakada

Department of Biotechnology,

Faculty of Engineering,

Kyoto Sangyo University,

Kamigamo-Motoyama,

Kita-ku, Kyoto 603-8555, Japan

T. Fukushige $\cdot$ T. Kanzaki

Department of Dermatology,

Faculty of Medicine,

Kagoshima University,

8-35-1 Sakuragaoka,

Kagoshima 890-8520, Japan deficiency is associated with the basic pathogenesis of this disease. To clarify the pathogenesis of this disease, we performed structural and immunocytochemical studies. The structure of human $\alpha$-NAGA deduced on homology modeling is composed of two domains, domain I, including the active site, and domain II. R329W/Q, identified in patients with Kanzaki disease have been deduced to cause drastic changes at the interface between domains I and II. The structural change caused by E325K found in patients with Schindler disease is localized on the N-terminal side of the tenth $\beta$-strand in domain II and is smaller than those caused by R329W/Q. Immunocytochemical analysis revealed that the main lysosomal accumulated material in cultured fibroblasts from patients with Kanzaki disease is Tn-antigen. These data suggest that a prototype of $\alpha$-NAGA deficiency in Kanzaki disease and factors other than the defect of $\alpha$-NAGA may contribute to severe neurological disorders, and Kanzaki disease is thought to be caused by a single enzyme deficiency.

Keywords $\alpha$ - $N$-acetylgalactosaminidase deficiency •

Schindler disease $\cdot$ Kanzaki disease $\cdot$ Homology modeling $\cdot$ Tn-antigen

\section{Introduction}

$\alpha$ - $N$-Acetylgalactosaminidase ( $\alpha$-NAGA) deficiency (Schindler/Kanzaki disease) is a genetic disease characterized by deficient activity of $\alpha$-NAGA (EC.3.2.1.49) and abnormal urinary excretion of glycopeptides (reviewed by Desnick and Schindler, 2001). Up to now, 12 patients from eight families with $\alpha$-NAGA deficiency have been reported, and this disease is known as a clinically heterogeneous disorder (Bakker et al. 2001; Kodama et al. 2001). In 1987, two German brothers with $\alpha$-NAGA deficiency were first described (van Diggelen et al. 1987). They developed an early-onset severe progressive neurological disorder pathologically characterized by an infantile neuroaxonal dystrophy 
(Schindler et al. 1989), and the disease was named Schindler disease. Then, a group of adult patients with angiokeratoma corporis diffusum and vacuolization in various cell types associated with $\alpha$-NAGA deficiency and glycopeptiduria identical to those in Schindler disease was reported (Kanzaki et al. 1989, 1991, 1993; Chabas et al. 1994; Kodama et al. 2001), and the disease was named Kanzaki disease. Then, $\alpha$-NAGA deficiency (Schindler/Kanzaki disease) was divided into three subtypes (types I-III) in a textbook (Desnick and Schindler, 2001). According to the latter classification, type I disease, an infantile neuroaxonal dystrophy associated with $\alpha$-NAGA deficiency, is an early-onset severe phenotype. Type II disease, angiokeratoma corporis diffusum associated with $\alpha$-NAGA deficiency but without overt neurological manifestations, is an adult-onset mild phenotype. Type III disease is an intermediate phenotype (de Jong et al. 1994).

However, there are two paradoxes. The first one is differences between the phenotype and genotype. Keulemans et al. (1996) pointed out that vacuolization, the hallmark of lysosomal storage diseases, was observed in various cells from type II patients but not in ones from type I patients. A pathological finding in the latter cases was 'spheroid degeneration' in axons, which does not indicate a lysosomal disease. Furthermore, they found that type I patients have some residual $\alpha$-NAGA activity, although the almost complete absence of $\alpha$-NAGA activity was found in type II patients (Keulemans et al. 1996). Two kinds of missense mutations (R329W and R329Q) and a nonsense mutation (E193X) were identified in Type II patients, each patient being homozygous for one of the mutations (Wang et al. 1994; Kodama et al. 2001; Keulemans et al. 1996). The E193X mutation identified in Spanish patients represents a null mutation that should be associated with a severe phenotype. But their phenotype is angiokeratoma corporis diffusum. On the other hand, two affected brothers with the type I phenotype are homozygous for E325K (Wang et al. 1990). So, the E325K mutation had been thought to be associated with a severe phenotype with an infantile neuroaxonal dystrophy. However, Bakker et al. (2001) recently reported two Moroccan sibs with $\alpha$-NAGA deficiency. They are homozygous for E325K alleles. The index patient, 3 years old, developed congenital cataracts and slight motor retardation, and his brother had no clinical or neurological symptoms at the age of 7 years, although it can be associated with the vegetative state of the first German brothers with Schindler disease. These data raise the question of whether or not an infantile neuroaxonal dystrophy really leads to the severe phenotype of $\alpha$-NAGA deficiency.

The second paradox is a difference between urinary glycopeptides identified in patients with $\alpha$-NAGA deficiency and a theoretical storage product. $\alpha$-NAGA is a glycosyl hydrolase that catalyzes the hydrolysis of Tn-antigen. Tn-antigen is a glycoprotein having a structure of GalNAc $\alpha 1-O-\mathrm{Ser} / \mathrm{Thr}$ and forms the core of $O$-linked glycoconjugates (Akita et al. 2001). It is also known as a tumor-associated antigen (Springer, 1984). A deficiency of the activity of $\alpha$-NAGA should lead to the accumulation of glycoconjugates with terminal $\alpha-N$ acetylgalactosaminyl residues. However, the major urinary excretion products are sialyl glycopeptides including NeuAc $\alpha 2-3 \mathrm{NeuAc} \beta \quad 1-3 \quad$ [NeuAc $\alpha 2-6$ ] GalNAc $\alpha 1-O-\operatorname{Ser} /$ Thr and NeuAc $\alpha 2-3$ Gal $\beta$ 1-3GalNAc $\alpha 1-O$-Ser/Thr-both in Schindler disease and Kanzaki disease (Linden et al. 1989; Hirabayashi et al. 1990). This discrepancy leads to the question of whether deficient activity of $\alpha$-NAGA is associated with the basic pathogenesis of this disease or not. It is very important to identify the major storage products in cells and tissues of patients with Schindler/Kanzaki disease to answer this question. But the details have not been clarified yet because there have been no autopsy cases of this disease.

In this study, we constructed a structural model of human $\alpha$-NAGA by homology modeling and then characterized the molecular defects in $\alpha$-NAGA caused by E325K and R329W/Q. Furthermore, we analyzed the accumulated materials in cultured fibroblasts from two patients with Kanzaki disease by means of immunocytochemical staining using an antibody against Tn-antigen and an antilysosomal-associated membrane protein-1 (LAMP-1) antibody, and lectin staining with a phytohemagglutinin purified from the seeds of Macckia amurensis (strongly mitogenic Macckia amurensis hemagglutinin, MAM), which is specific for NeuAc $\alpha 2-3 \mathrm{Gal}$ (Kawaguchi et al. 1974). LAMP-1 is a lysosomal membrane protein. It is involved in protecting the lysosomal membrane from autodigestion and its expression is increased in lysosomal diseases (Hua et al. 1997; Meikle et al. 1998). In this study, LAMP-1 was used as a lysosomal marker. The pathogenesis of $\alpha$-NAGA deficiency is discussed.

\section{Materials and methods}

Structural modeling of human wild-type and mutant $\alpha$-NAGAs

Structural models of human wild-type $\alpha$-NAGA and its mutants (E325K, R329W and R329Q) were built based on crystallographic structure data for chicken $\alpha$-NAGA (PDB code; 1KTB) (Garman et al. 2002) exhibiting $75 \%$ amino acid identity. The modeling was performed with molecular modeling software, SYBYL/ COMPOSER and BIOPOLIMER (TRIPOS, Inc., Mountain View, CA, USA). The sequence of human $\alpha$-NAGA was aligned with that of chicken $\alpha$-NAGA. According to the sequence alignment, the backbone structure of chicken $\alpha$-NAGA was applied to the model of human $\alpha$-NAGA. After each side chain had been generated and adjusted, the energy minimization procedure was performed to optimize the conformations and side-chain rotamers, as described previously (Sakuraba et al. 2002). The mutant models were built in the same way as for the wild-type but were based on the amino acid sequence with the replacements. To determine the influence of amino acid substitutions on the model structure, each mutant model was superimposed with the wild-type model based on the $\mathrm{C} \alpha$ atoms by a least-square-mean fitting method (Kabsh, 1976, 1978).

We defined the structure as being influenced by the amino acid replacement when the position of an atom of the mutant differed from that of the wild type by more than the total root-mean-square distance value, as described previously (Sakuraba H et al. 2002). 
Immunocytochemical staining

Mouse monoclonal antibodies to LAMP-1 and Tn-antigen were purchased from Southern Biotechnology (Birmingham, AL, USA) and DAKO (Glostrup, Denmark), respectively. A fluorescein isothiocyanate (FITC)-conjugated MAM was purchased from Seikagaku Co. (Tokyo, Japan). FITC-conjugated goat antimouse $\operatorname{IgM}\left(\mathrm{Fab}^{\prime}\right)_{2}$ and Cy3-conjugated goat antimouse $\operatorname{IgG}(\mathrm{Fab})_{2}$ were purchased from Jackson Immuno Research (West Grove, PA, USA).

Cultured skin fibroblasts from two unrelated Japanese patients with Kanzaki disease, a patient with sialidosis (lysosomal sialidase deficiency) as a pathological control, and normal control subjects were cultured in Ham's F-10 medium supplemented with 10\% fetal calf serum and antibiotics at $37^{\circ} \mathrm{C}$ in a humidified incubator flushed continuously with a $5 \% \mathrm{CO}_{2}-95 \%$ air mixture.

For the double staining of Tn-antigen and LAMP-1, cells grown on Lab-Tek chamber slides (Nunc, Naperville, IL, USA) were fixed with $4 \%$ paraformaldehyde in phosphate-buffered saline (PBS), pH7.4, for $5 \mathrm{~min}$, followed by blocking with $1 \%$ bovine serum albumin in PBS for $30 \mathrm{~min}$ at room temperature. The cells were then incubated for $1 \mathrm{~h}$ with a mouse monoclonal antibody to Tn-antigen (1:100 diluted) in a dark place. After washing, the cells were incubated for $1 \mathrm{~h}$ with FITC-conjugated goat antimouse IgM $\left(\mathrm{Fab}^{\prime}\right)_{2}(1: 100$ diluted). Then the cells were washed and incubated for $1 \mathrm{~h}$ with a mouse monoclonal antibody to LAMP-1 (1:100 diluted) in a dark place. After washing, the cells were reacted for $1 \mathrm{~h}$ with Cy3-conjugated goat antimouse IgG (Fab') $)_{2} \quad(1: 200$ diluted) in a dark place. The stained cells were examined under a microscope (Axiovert 100 M; Carl Zeiss, Oberkochen, Germany) equipped with a confocal laser scanning imaging system (LSM510; Carl Zeiss).

The double staining of cultured fibroblasts with FITC-MAM and an anti-LAMP-1 antibody was performed according to the modified method of Kotani et al. (submitted). Briefly, the cells were reacted for $1 \mathrm{~h}$ with a FITC-conjugated MAM (1:100 diluted). After washing, the cells were incubated for $1 \mathrm{~h}$ with an anti-LAMP-1 antibody (1:100 diluted) as the first antibody in a dark place. After washing, the cells were reacted for $1 \mathrm{~h}$ with Cy3-conjugated goat antimouse $\operatorname{IgG}(\text { Fab') })_{2}(1: 200$ diluted $)$ as the second antibody in a dark place.

Establishment of cultured fibroblasts was performed with informed consent from the patients, and the study was approved by the ethical committees of our institutions.

\section{Results}

Structural modeling of human wild-type and mutant $\alpha$-NAGAs

We built a model of human $\alpha$-NAGA based on the crystallographic structural data for chicken $\alpha$-NAGA without the $\mathrm{N}$-terminal 17 residues corresponding to the signal peptide. The structure corresponding to the C-terminal 7 residues has not been determined for the chicken $\alpha$-NAGA structure. The model, thus, comprises 387 amino acids (i.e., residues 18-404). The modeled structure of human $\alpha$-NAGA is shown in Fig. 1. The structure of human $\alpha$-NAGA has two domains (domains I and II). Domain I (residues 18-311) is folded into a $(\beta / \alpha)_{8}$-barrel with the active site (D115 and D217), and domain II (residues 312-404) has a $\beta$ sandwich fold consisting of eight antiparallel $\beta$-strands. To determine the positions of mutations, amino acid residues E325 and R329 were mapped on the monomer and dimer structures of wild-type human $\alpha$-NAGA (Figs. 1 and 2). As shown in Fig. 1, the residues of E325 and R329 are located on opposite sides of the same tenth $\beta$-strand in domain II. The dimer model indicates that both E325 and R329 are not concerned with the dimer interface (Fig. 2).

To reveal the influence of the mutations, we constructed three mutant structures (R329W, R329Q and $\mathrm{E} 325 \mathrm{~K}$ ) and compared them with the wild-type. R329 is located on the $\mathrm{C}$-terminal side of the tenth $\beta$-strand in domain II, facing domain I (Fig. 1). In the wild-type model, the side chain of R329 fits into the domain I and forms hydrogen bonds with three residues; T274 and N306 in domain I, and I312 at the junction between the two domains (Fig. 3A). These hydrogen bonds between domains I and II are supposed to the stability of the two domains. Substitutions of R329W and R329Q cause disruption of these hydrogen bonds. In addition to the loss of the hydrogen bonds, R329W introduces a bulkier side chain that results in steric clashes. As shown in Fig. 3B and C, R329W/Q substitutions cause a drastic structural change on the interface between domains I and II. E325 is located on the N-terminal side of the tenth $\beta$-strand in domain II. The side chain is surrounded by charged residues (E234, R316, and K319) and polar residues (N230, Y327, and C343). Substitution of E325 with a positively charged $\mathrm{K}$ residue causes alteration of the arrangement of these residues and conformational changes of their side chains (Fig. 4). The predicted structural change is smaller than those caused by $\mathrm{R} 329 \mathrm{~W} / \mathrm{Q}$.

Immunocytochemical staining of cultured fibroblasts from patients with Kanzaki disease

To identify storage materials in cultured fibroblasts from Kanzaki disease patients, we tried double staining of cultured fibroblasts with an anti-Tn-antigen antibody and an anti-LAMP-1 antibody or with MAM and an anti-LAMP-1 antibody. We first performed double staining using an antibody against Tn-antigen and an anti-LAMP-1 antibody. As shown in Fig. 5, staining with the anti-LAMP-1 antibody revealed granular fluorescence, indicating lysosomes. When cells were stained with the anti-LAMP-1 antibody, both sialidosis fibroblasts and Kanzaki disease fibroblasts showed strong fluorescence, although normal controls showed weak granular fluorescence. This suggests an activated lysosomal function in these diseases. Marked fluorescence was only found in Kanzaki disease fibroblasts when the antibody against $\mathrm{Tn}$-antigen was used. The localization was almost identical to that of LAMP-1 (Fig. 5A). However, only very weak fluorescence was detected in Kanzaki disease fibroblasts or in normal subjects when the cells were stained with MAM, although strong fluorescence was found in sialidosis fibroblasts in which sialylated glycoconjugates should be accumulated (Fig. 5B). The results were confirmed by three independent experiments. These results suggest that the 
accumulated material in Kanzaki disease cells is Tn-antigen and not a sialyl glycoconjugate.

\section{Discussion}

$\alpha$-NAGA deficiency has been shown to be a clinically and pathologically heterogeneous disease including Schindler disease and Kanzaki disease. We tried to gain an insight into the pathogenesis of $\alpha$-NAGA deficiency on the basis of structural information. The modeled human $\alpha$-NAGA consists of two domains, one of which has a $(\beta / \alpha)_{8}$-barrel structure including the active site and the other an $\alpha / \beta$ topology. This structure may be common in many lysosomal hydrolases (Mark et al. 2003). A previous study (Wang et al. 1990) demonstrated that $\alpha$-NAGA formed a homodimer of a $48-\mathrm{kDa}$ species in cultured fibroblasts. Structural analysis revealed that E325 and R329 are located on the N-terminal and C-terminal sides of the tenth $\beta$-strand in domain II, respectively. E325 and R329 are far from the dimer interface, and both are thus thought not to affect the dimer formation.

Among the three missense mutations (R329W, $\mathrm{R} 329 \mathrm{Q}$, and $\mathrm{E} 325 \mathrm{~K}$ ), R329W and R329Q identified in patients with Kanzaki disease are deduced to cause drastic structural changes on the interface between domains I and II. The hydrogen bonds that R329 forms between domains I and II are supposed to contribute to the stability of the two domains. The R329W/Q substitutions causing the loss of these hydrogen bonds could destabilize the protein structure. Furthermore, the structural changes on the interface between the two

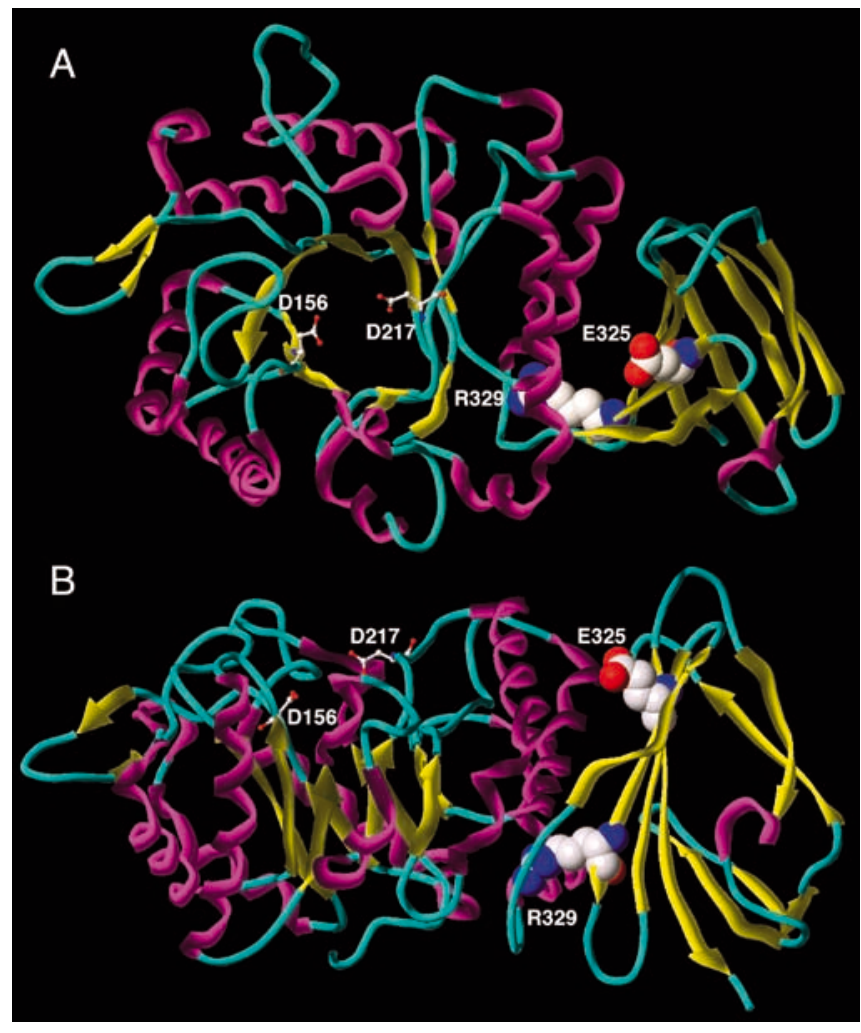

Fig. 1A, B Three-dimensional structure of human $\alpha$-NAGA. A Front view of human $\alpha$-NAGA. B Side view of the protein. The secondary structures are shown as tube and ribbon drawings; $\alpha$-helix (magenta), $\beta$-sheet (yellow), and coil (cyan). Residues comprising the catalytic site (D156 and D217) are presented as a ball-and-stick model, and the localization of amino acid substitutions (E325 and R329) is presented as a space-filling model
Fig. 2 Deduced structure of the dimer of human $\alpha$-NAGA

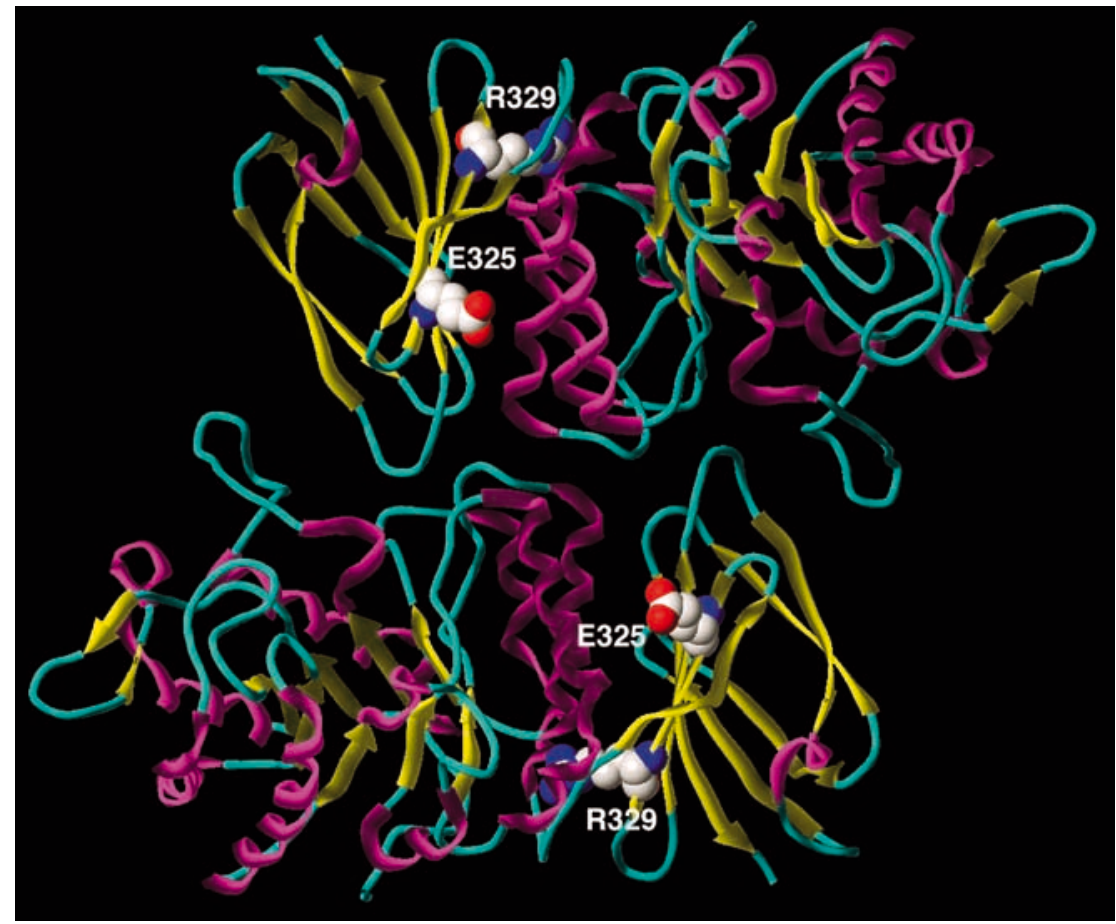



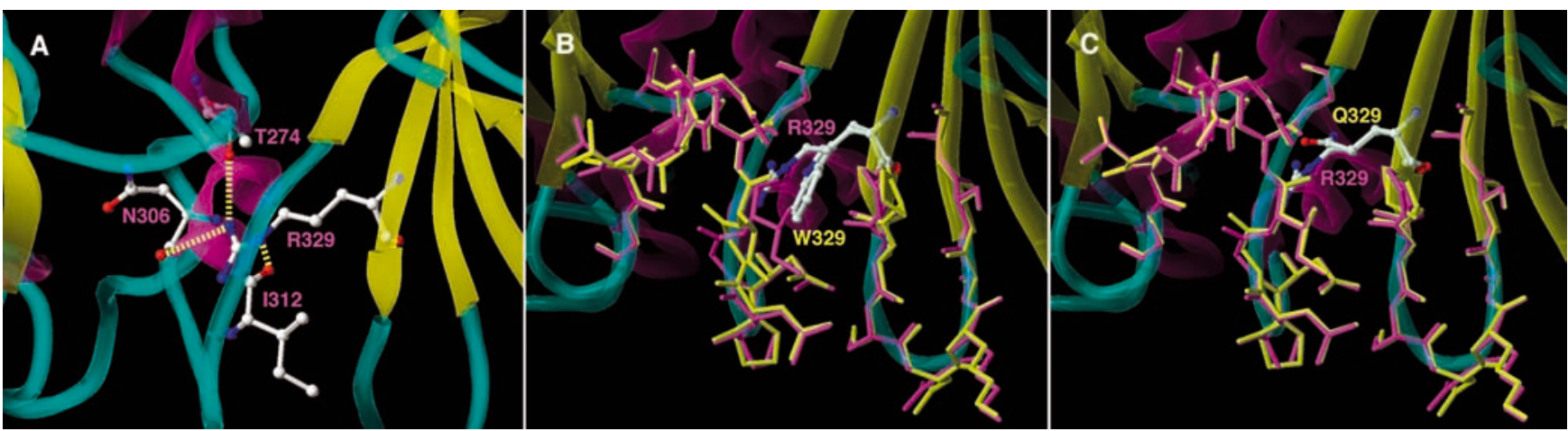

Fig. 3A-C Conformational changes of $\alpha$-NAGA caused by R329W/Q. A Hydrogen bonds between R329 and other amino acid residues. B Structural change caused by R329W. C Structural change caused by $\mathrm{R} 329 \mathrm{Q}$. The residues of the wild type and mutants are colored magenta and yellow, respectively

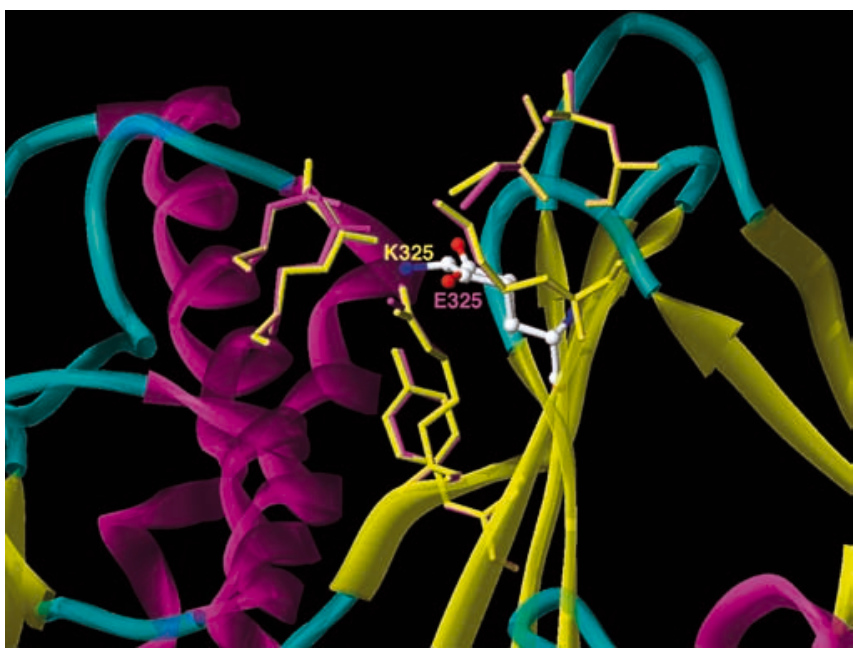

Fig. 4 Conformational changes of $\alpha$-NAGA caused by E325K. The residues of the wild type and mutant are colored magenta and yellow, respectively

domains would affect the packing of the protein. These structural changes were observed not only on side chains but also on main chains forming the domain structure. Thus, the substitutions are deduced to cause a significant influence to decrease the protein stability or to cause a folding defect. $\alpha$-NAGA activities in clinical samples from the patients homozygous for these mutations are both very low (below $1 \%$ of the normal control mean) (Kanzaki et al. 1991; Kodama et al. 2001), and the kinetic data could not be obtained. However, R329 is far from the active site, and it is thought not to affect the kinetic character, including $\mathrm{Km}$ value. Indeed, Kanzaki et al. performed immunoblotting analysis and found that no band representing the mature form of $\alpha$-NAGA was detected in a patient with the R329W mutation (Kanzaki et al. 1991).

On the other hand, the deduced structural change caused by E325K was localized on the $\mathrm{N}$-terminal side of the tenth $\beta$-strand and observed on only side-chain conformations.
Thus, the influence caused by $\mathrm{E} 325 \mathrm{~K}$ is deduced to be smaller than that caused by R329W/Q. Keulemans et al. (1996) reported that the synthesis of precursor $\alpha$-NAGA was normal, but mature $\alpha$-NAGA was not detectable with the $\mathrm{E} 325 \mathrm{~K}$ mutation, and the $\alpha$-NAGA activities in patients homozygous for $\mathrm{E} 325 \mathrm{~K}$ are relatively higher (0.6-1.7\% of the normal control mean) than those in Spanish Kanzaki patients homozygous for E193X (0.2\% of the normal control mean). We could not obtain clinical samples from patients with Schindler disease and the kinatic data are not available. But, E325 is far from the active site. So, the structural change caused by E325K is thought to cause the expression of an unstable enzyme and the expressed protein would be degraded quickly.

Our structural data are compatible with those of comparative studies including clinical, biochemical, pathological, and genetic analyses (Keulemans et al. 1996; Bakker et al. 2001), and the results support the suggestion of Keulemans et al. (1996) and Bakker et al. (2001) that a complete $\alpha$-NAGA deficiency would cause late-onset angiokeratoma corporis diffusum (Kanzaki disease), and factors other than the defect of $\alpha$-NAGA may contribute to the phenotypic expression in patients with an infantile neuroaxonal dystrophy (Schindler disease). The fact that patients other than the first German sibs with the combination of $\alpha$-NAGA deficiency and neuroaxonal dystrophy have not been found would support this, although extensive research to find further patients has been performed.

In $\alpha$-NAGA deficiency, the main urinary excretion products (sialyl glycoconjugates) are different from a theoretical accumulation material (Tn-antigen) resulting from a defect of $\alpha$-NAGA. A possible explanation is that the accumulated Tn-antigen in various kinds of cells is probably taken up from the blood by hepatocytes in the liver, where glycosyltransferases, including $\alpha 2-6$ sialyltransferase, are abundant, and the addition of galactose residues and $N$-acetylneuraminic acid residues could occur. As a result, excessive sialyl glycoconjugates produced in the liver would be carried to the kidneys and excreted in the urine.

To investigate the primary accumulated materials in cells, we performed immunocytochemical and lectin staining using cultured fibroblasts as the best samples because they are not influenced by the metabolism in the liver via blood. Unfortunately, we could not obtain 
Fig. 5A, B Immunocytochemical analysis of LAMP-1 and Tn-antigen, and lectin staining with MAM using cultured fibroblasts as samples. A Double staining with an antiLAMP-1 antibody (red) and an antibody against $\mathrm{Tn}$-antigen (green). Lamp-1, staining with the anti-LAMP-1 antibody; Tn, staining with the antibody against Tn-antigen; Merge, double staining with these materials; Phase contrast, a phase contrast figure. B Double staining with the anti-LAMP-1 antibody (red) and MAM (green). Lamp-1, staining with the anti-LAMP-1 antibody; MAM, lectin staining with MAM; Merge, double staining with these materials; Phase contrast, a phase contrast figure. Kanzaki 631 and Kanzaki 632, unrelated patients with Kanzaki disease; Sialidosis, a patient with sialidosis; Control, a normal subject
A

LAMP-1
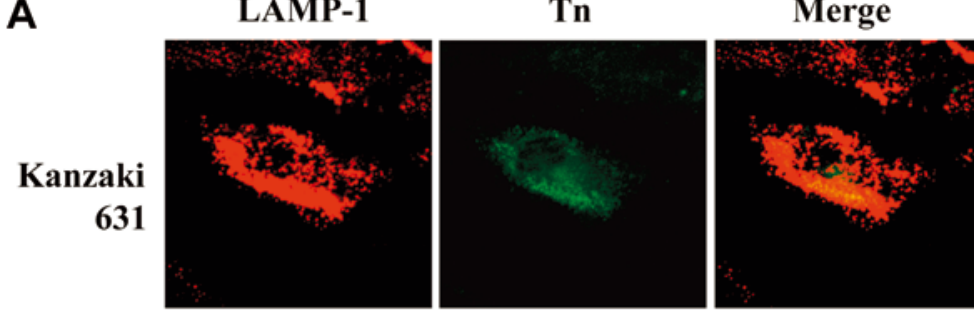

Phase contrast
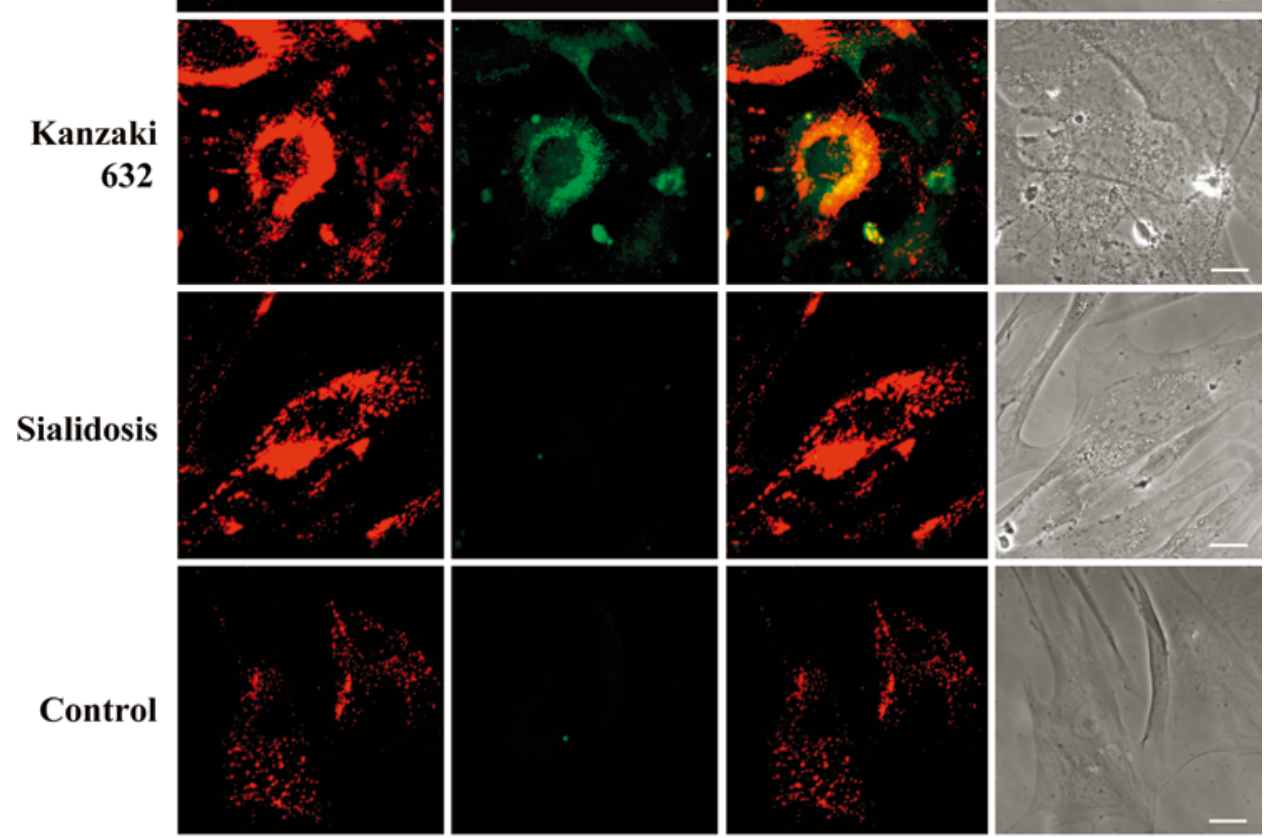

B
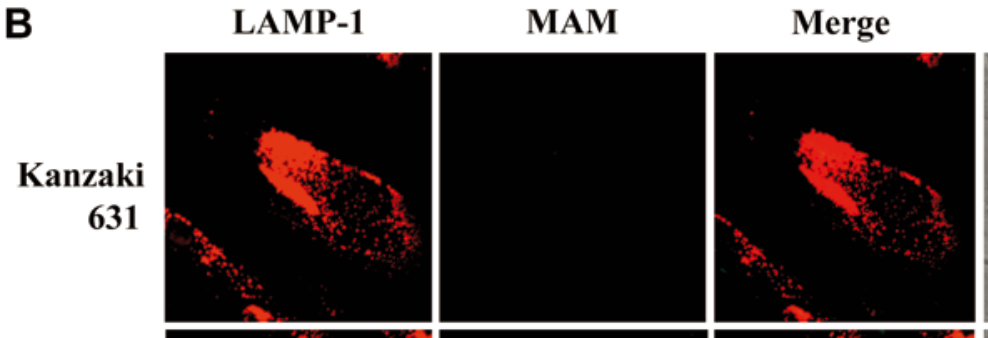

Phase contrast

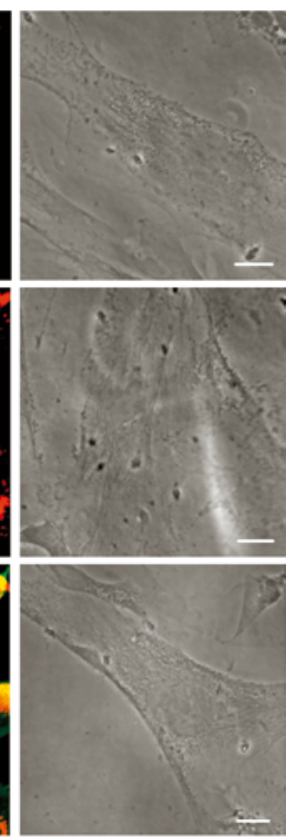

Sialidosis
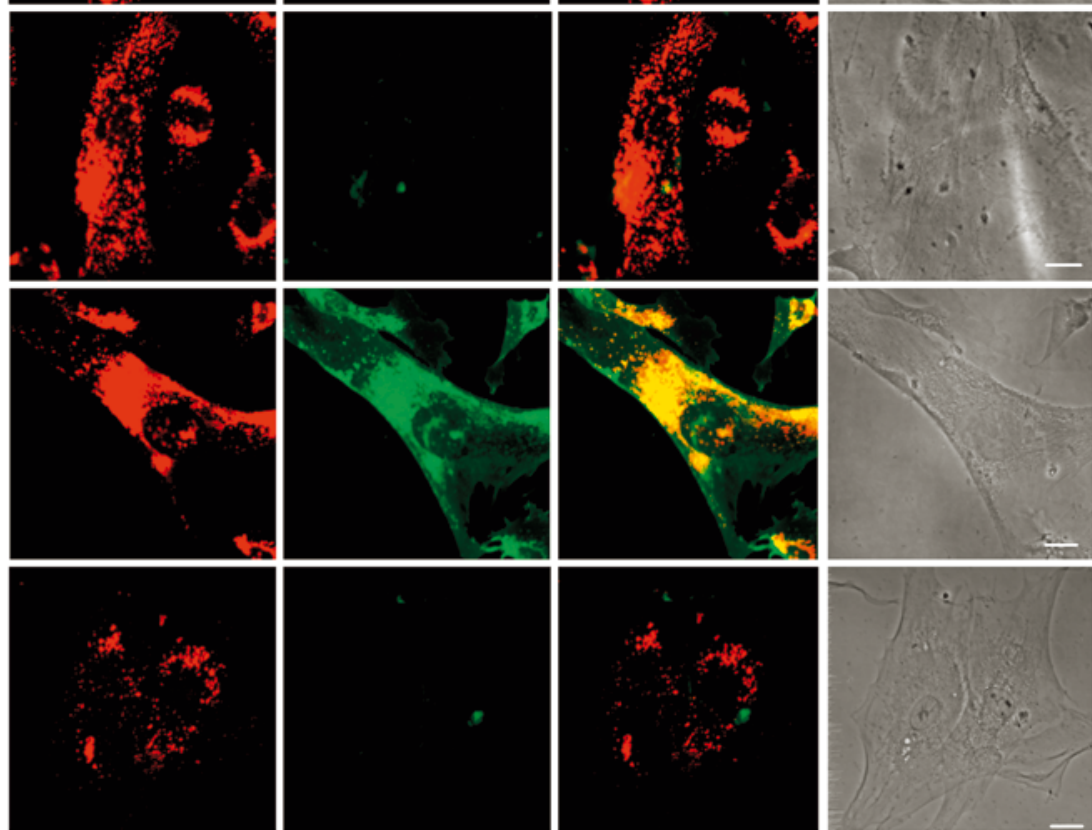
fibroblasts from patients with Schindler disease. However, considering the data including those of previously reported biochemical and pathological analyses and these structural analyses, Kanzaki disease seems to be a prototype of $\alpha$-NAGA deficiency. So, we used cultured fibroblasts from patients with Kanzaki disease as samples for the investigation of intracellular storage materials. The sensitive immunocytochemical analyses revealed that $\mathrm{Tn}$-antigen is the major storage product. We also tried an immunoblotting analysis of Tn-antigen but could find no suitable antibodies available for that. However, Kanda et al. (2002) demonstrated that Tn-antigen was accumulated mainly in lysosomes of vascular endothelial cells, eccrine sweat gland cells, fibroblasts, and pericytes in Kanzaki disease patients using biopsied skin specimens by means of immunoelectron microscopic analyses. Considering these results, Kanzaki disease is thought to result from the defect of $\alpha$-NAGA deficiency.

In conclusion, we characterized the structural and immunochemical bases of $\alpha$-NAGA deficiency. The structural change caused by $\mathrm{R} 329 \mathrm{~W} / \mathrm{Q}$ is greater than that by $\mathrm{E} 325 \mathrm{~K}$. The main intracellular accumulated product in this disease is Tn-antigen. These findings are useful for understanding the pathogenesis of $\alpha$-NAGA deficiency.

Acknowledgements This work was partly supported by grants from the Tokyo Metropolitan Government, The Japan Society for the Promotion of Science, and the Ministry of Health, Labor and Welfare of Japan.

\section{References}

Akita K, Fushiki S, Fujimoto T, Inoue M, Oguri K, Okayama M, Yamashina I, Nakada H (2001) Developmental expression of a unique carbohydrate antigen, Tn antigen, in mouse central nervous tissues. J Neurosci Res 65:595-603

Bakker HD, de Sonnaville M-LCS, Vreken P, Abeling NGGM, Groener JEM, Keulemans JLM, van Diggelen OP (2001) Human $\alpha$ - $N$-acetylgalactosaminidase ( $\alpha$-NAGA) deficiency: no association with neuroaxonal dystrophy? Eur J Hum Genet 9:91-96

Chabas A, Coll MJ, Aparicio M, Rodriguez Diaz E (1994) Mild phenotype expression of $\alpha$ - $N$-acetylgalactosaminidase deficiency in two adult siblings. J Inher Metab Dis 17:724-731

de Jong J, van den Berg C, Wijburg $\mathrm{H}$, Willemsen $\mathrm{R}$, van Diggelen O, Schindler D, Hoevenaars F, Wevers R (1994) Alpha- $N$-acetylgalactosaminidase deficiency with mild clinical manifestations and difficult biochemical diagnosis. J Pediatr 125:385-391

Desnick RJ, Schindler D (2001) $\alpha$-N-Acetylgalactosaminidase deficiency: Schindler disease. In: Scriver CR, Beaudet AL, Sly WS, Valle D (eds) The metabolic and molecular bases of inherited disease, 8th edn. McGraw-Hill, New York, pp. 34833505

Garman SC, Hannick L, Zhu A, Garboczi DN (2002) The $1.9 \AA$ structure of $\alpha-N$-acetylgalactosaminidase: molecular basis of glycosidase deficiency diseases. Structure 10:425-434

Hirabayashi Y, Matsumoto Y, Matsumoto M, Toida T, Iida N, Matsubara T, Kanzaki T, Yokota M, Ishizuka I (1990) Isolation and characterization of major urinary amino acid $O$-glycosides and a dipeptide $O$-glycoside from a new lysosomal storage disorder (Kanzaki disease). J Biol Chem 265:1693-1701
Hua CT, Hopwood JJ, Carlsson SR, Harris RY, Meikle PJ (1998) Evaluation of the lysosome-associated membrane protein LAMP-2 as a maker for lysosomal storage disorders. Clin Chem 44:2094-2102

Kabsh W (1976) A solution for the best rotation to relate two sets of vectors. Acta Crystallogr A32:922-923

Kabsh W (1978) A discussion of the solution for the best rotation to relate two sets of vectors. Acta Crystallogr A34:827-828

Kanda A, Tsuyama S, Murata F, Kodama K, Hirabayashi Y, Kanzaki T (2002) Immunoelectron microscopic analysis of lysosomal deposits in $\alpha$ - $N$-acetylgalactosaminidase deficiency with angiokeratoma corporis diffusum. J Dermatol Sci 29:42-48

Kanzaki T, Yokota M, Mizuno N, Matsumoto Y, Hirabayashi Y (1989) Novel lysosomal glycoaminoacid storage disease with angiokeratoma corporis diffusum. Lancet $\mathrm{i}: 875-876$

Kanzaki T, Wang AM, Desnick RJ (1991) Lysosomal $\alpha-N$-acetylgalactosaminidase deficiency, the enzymatic defect in angiokeratoma corporis diffusum with glycopeptiduria. J Clin Invest 88:707-711

Kanzaki T, Michino Y, Fumitoshi I, Hirabayashi Y, Wang AM, Desnick RJ (1993) Angiokeratoma corporis deffusum with glycopeptiduria due to deficient lysosomal $\alpha$ - $N$-acetylgalactosaminidase activity. Arch Dermatol 129:460-466

Kawaguchi T, Matsumoto I, Osawa T (1974) Studies on hemagglutinin from Macckia amurensis seeds. J Biol Chem 249:27862792

Keulemans JLM, Reuser AJJ, Kroos MA, Willemsen R, Hermans MMP, van den Ouweland AHW, de Jong JGN, Wevers RA, Renier WO, Schindler D, Coll MJ, Chabas A, Sakuraba H, Suzuki Y, van Diggelen OP (1996) Human $\alpha$ - $N$-acetylgalactosaminidase $(\alpha$-NAGA) deficiency: new mutations and the paradox between genotype and phenotype. J Med Genet 33:458-464

Kodama K, Kobayashi H, Abe R, Ohkawa A, Yoshii N, Yotsumoto S, Fukushige T, Nagatsuka Y, Hirabayashi Y, Kanzaki T (2001) A new case of $\alpha-N$-acetylgalactosaminidase deficiency with angiokeratoma corporis diffusum, with Menier's sydrome and without mental retardation. Br J Dermat 144:363-368

Linden HU, Klein RA, Egge H, Peter-Katalinic J, Dabrowski J, Schindler D (1989) Isolation and structural characterization of sialic acid-containing glycopeptides of the $O$-glycosidic type from the urine of two patients with a hereditary deficiency in $\alpha-N$-acetylgalactosaminidase activity. Biol Chem Hoppe-Seyler 370:661-672

Mark BL, Mahuran DJ, Cherney MM, Zhao D, Knapp S, James MNG (2003) Crystal structure of human $\beta$-hexosaminidase B: Understanding the molecular basis of Sandhoff and Tay-Sachs disease. J Mol Biol 327:1093-1109

Meikle RJ, Brooks DA, Ravenscroft EM, Yan M, Williams RE, Jaunzems AE, Chataway TK, Karageorgos LE, Davey RC, Boulter CD, Carlsson SR, Hopwood JJ (1997) Diagnosis of lysosomal storage disorders: evaluation of lysosome-associated membrane protein LAMP-1 as a diagnostic marker. Clin Chem 43:1325-1335

Sakuraba H, Matsuzawa F, Aikawa S, Doi H, Kotani M, Lin H, Ohno K, Tanaka A, Yamada H, Uyama E (2002) Molecular and structural studies of the GM2 gangliosidosis 0 variant. J Hum Genet 47:176-183

Schindler D, Bishop DF, Wolfe DE, Wang AM, Egge H, Lemieux RU, Desnick RJ (1989) Neuroaxonal dystrophy due to lysosomal $\alpha-N$-acetylgalactosaminidase deficiency. N Engl J Med 320:1735-1740

Springer GF (1984) T and Tn, general carcinoma antigens. Science 224:1198-1206

van Diggelen OP, Schindler D, Kleijer WJ, Huijmans JMG, Galjaard H, Linden HU, Peter-Katalinic J, Egge H, Dabrowski U, Cantz M (1987) Lysosomal $\alpha-N$-acetylgalactosaminidase deficiency: a new inherited metabolic disease. Lancet 2:804

Wang AM, Bishop DF, Desnick RJ (1990) Human $\alpha-N$-acetylgalactosaminidase: molecular cloning, nucleotide sequence, and expression of a full-length cDNA. J Biol Chem 265:2185921866 
Wang AM, Schindler D, Desnick (1990) Schindler disease: the molecular lesion in the $\alpha-N$-acetylgalactosaminidase gene that causes an infantile neuroaxonal dystrophy. J Clin Invest $86: 1752-1756$
Wang AM, Kanzaki T, Desnick RJ (1994) The molecular lesion in the $\alpha-N$-acetylgalactosaminidase gene that causes angiokeratoma corporis diffusum with glycopeptiduria. J Clin Invest 94:839-845 